\section{Cureus}

\title{
Correlation of Foraminal Area and Response to Cervical Nerve Root Injections
}

\author{
Wilson Z. Ray ${ }^{1}$, Syed Akbari ${ }^{2}$, Lubdha M. Shah ${ }^{3}$, Erica Bisson ${ }^{4}$ \\ 1. Neurological Surgery, Washington University School of Medicine 2. School of Medicine, Washington \\ University School of Medicine 3. Department of Radiology, University of Utah 4. Department of \\ Neurosurgery, University of Utah
}

$\square$ Corresponding author: Erica Bisson, erica.bisson@hsc.utah.edu Disclosures can be found in Additional Information at the end of the article

\section{Abstract}

Introduction: Patients with age-related degenerative changes in the cervical spine leading to cervical spondylosis may be symptomatic or asymptomatic. Older patients with radicular pain tend to have a better response to epidural steroid injections, but it is often difficult to predict which patients will have a positive response to selective nerve root block (SNRB). We analyzed whether the cervical neuroforaminal area measured on MRI predicts immediate therapeutic responses to SNRB in patients who have cervical radiculopathy.

Methods: We retrospectively reviewed all patients who had cervical SNRBs treated at a single tertiary referral center. We recorded patient demographics, the neuroforaminal area of the symptomatic and contralateral sides, Visual Analog Scale (VAS) score pre- and post-injection, history of previous cervical surgery, comorbidities, and history of tobacco use.

Results: Sixty-four patients with symptoms of cervical radiculopathy treated with neuroforaminal nerve root injections had appropriate imaging and VAS scores recorded. The average foraminal area of the symptomatic side before treatment was significantly smaller than the contralateral asymptomatic neuroforamen $(p<0.0001)$. Those patients with the smallest neuroforamen had a positive response to SNRB. Diabetes and tobacco use did not influence patient response to treatment.

Conclusions: Measurement of neuroforaminal areas on MRI may represent a useful preprocedural technique to predict which patients with symptoms of cervical radiculopathy secondary to foraminal stenosis are likely to respond to selective nerve root injections. The predictive ability appears to be limited to those patients with severe stenosis and was less useful in those patients with moderate or mild stenosis.

Received 04/02/2015

Review began 04/04/2015

Review ended 07/06/2015

Published 07/20/2015

(c) Copyright 2015

Ray et al. This is an open access article distributed under the terms of the Creative Commons Attribution License CC-BY 3.0., which permits unrestricted use, distribution, and reproduction in any medium, provided the original author and source are credited.
Categories: Radiology, Neurosurgery

Keywords: cervical spine, selective nerve root block, neuroforamen, cervical radiculopathy, pain, stenosis

\section{Introduction}

Age-related degenerative changes in the cervical spine leading to cervical spondylosis are commonly observed in both symptomatic and asymptomatic patients. Spondylotic changes can lead to a broad range of overlapping symptoms, including axial neck pain, cervical radiculopathy, cervical myelopathy, or myeloradiculopathy, although corresponding changes on magnetic resonance imaging (MRI) do not always exist. Currently at our institution, some 
patients with symptomatic spondylotic changes are initially treated with a diagnostic/therapeutic selective nerve root block (SNRB). While many patients have a very good response to the injection, there remains a large subset of patients that, despite correlating MRI and clinical findings, fail to have a positive response to the injection. The efficacy of lumbar SNRBs remains unconfirmed, with mixed reports in the literature [1-2], and studies investigating the efficacy of cervical nerve root injections and parameters that influence response to injection are scarce.

Previous work has suggested older patients with radicular pain tend to have a better response to both transforaminal and interlaminar cervical epidural steroid injections [3-5], but it is often difficult to predict which patients will have a positive response to SNRB. A surgeon's personal experience and the characteristics of the patient's spine on MRI (e.g., hard disc, soft disc, osteophytes) also weigh significantly on who is referred for nerve root injection, and little literature exists regarding the role of the neuroforaminal area in clinical response. We hypothesized that patients with foraminal areas below a critical threshold in size would not have a good response to injection because of limited anesthetic/steroid penetration. The purpose of this study was to assess whether the cervical neuroforaminal area measured on MRI can be used to predict immediate therapeutic responses to cervical SNRB in patients with symptoms of cervical radiculopathy.

\section{Materials And Methods}

After receiving approval from the University of Utah Institutional Review Board (protocol \#53330) with a waiver of informed consent, we undertook a retrospective review of patients who had undergone a cervical SNRB over a two-year period (January, 2009-December, 2010) treated at our tertiary referral center. Inclusion/exclusion criteria included patients referred to our department for surgical evaluation of symptoms of cervical radiculopathy (motor, sensory, or pain), an MRI $\leqslant 6$ months old available for direct review, no findings of cervical myelopathy, and no history of previous cervical surgery. All patients had a clinical history of cervical radiculopathy with MRI findings corresponding to physical examination findings. No patients with cervical myelopathy or purely axial neck pain were included in the analysis. Only patients receiving injections for a single level were included. We collected patient demographics, the Visual Analog Scale (VAS) score pre- and post-injection, history of previous cervical surgery, comorbidities, and history of tobacco use.

All SNRBs were done with fluoroscopic guidance using a biplane fluoroscopic unit. The patient was placed in the supine position. The vertebral and facet joint margins were aligned under fluoroscopy. The target was the superior articular process of the same number vertebra as the nerve being injected. Approximately $1 \mathrm{~mL}$ of $2 \%$ lidocaine was used for local analgesia. The needle was then deflected anteriorly into the neural foramen. Approximately $2 \mathrm{~mL}$ of myelography-safe iodinated contrast agent was used for the needle tip confirmation in the nerve root sleeve. The contrast pattern was identified as intraneural, perineural, and, most importantly, not intra-arterial. The injectate used in these procedures was $1 \mathrm{~mL}$ of $10 \mathrm{mg} / \mathrm{mL}$ dexamethasone and $1 \mathrm{~mL}$ of $1 \%$ lidocaine. We used nonparticulate steroids (dexamethasone) for our neural foraminal injections because of the reported risk of embolic infarctions due to inadvertent intra-arterial injection of particulate steroids [6-7]. A post-procedure VAS score was obtained 30 minutes after the injection by the treating neurointerventionalist.

We measured the neuroforaminal area of the symptomatic side, using the asymptomatic contralateral side as the internal control. Measurements of neuroforaminal areas were made by both a spine neurosurgeon and a neuroradiologist blinded to patient VAS scores. Measurements were performed on the MRI Digital Imaging and Communications in Medicine (DICOM) data set, imported into the Osirix software (version 3.8.1 32-bit). The sagittal T2-weighted sequences were selected and post-processed with the three-dimensional multi-projection 


\section{Cureus}

reformat program. Using the crosshairs, maximal neuroforaminal dimensions were evaluated at the level of interest in the oblique sagittal plane by each observer independently. The region of interest tool was used to calculate the maximum dimension of the neural foramen in the oblique sagittal plane (Figure 1).

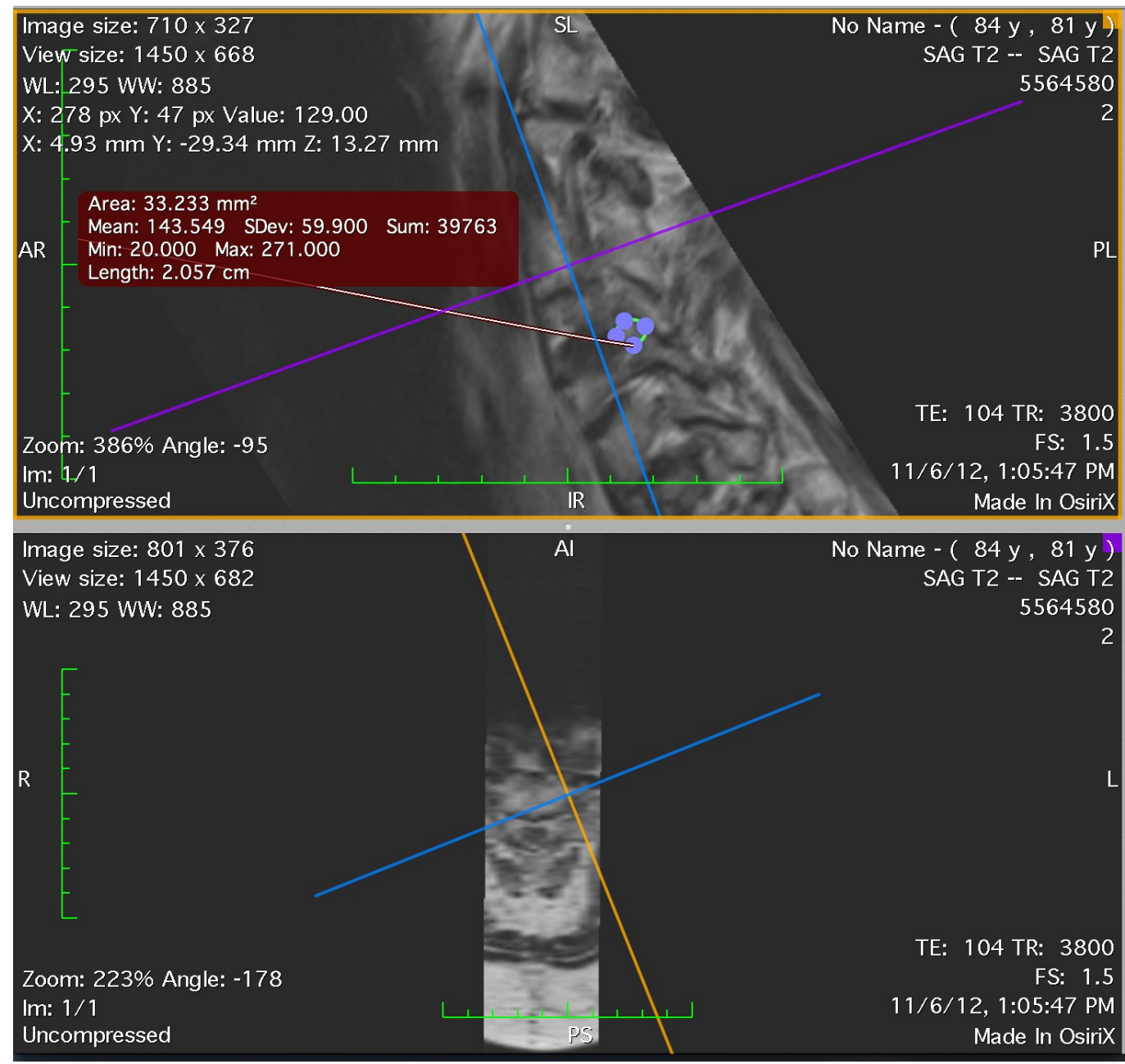

FIGURE 1: The region of interest tool was used to calculate the maximum dimension of the neural foramen in the oblique sagittal plane.

\section{Statistical analysis}

Statistical analyses were performed using a Student $t$-test for paired ipsilateral and contralateral foraminal area. Analyses involving continuous variables across four groups were performed using ANCOVA, with diabetes and smoking status as covariates. The analyses were performed using SPSS. All tests were two-sided, and a P-value $<0.05$ was used to indicate statistical significance.

\section{Results}

Sixty-four patients were identified for inclusion in this study. The average age of the subjects in the cohort was $48.1 \pm 8.9$ years (range: $24-70$ years). The distribution by sex was approximately equal (Table 1). Twenty percent of patients were current or former smokers. A small minority of 


\section{Cureus}

patients were diabetic (6.3\%), with the most common comorbidity being hypertension (15\%). The most commonly treated level was C5/6 (29 patients, 45.3\%); 24 (37.5\%) patients were treated at C6/7, and the number of patients treated at other levels was much lower.

$\mathbf{N}=64(\%)$

Sex

Female

$31(48.4)$

Male

$33(51.6)$

Number of patients treated at each spinal level (\%)

C3-4

C4-5

C5-6

C6-7

$24(37.5)$

C7-T1

Smoking status

Current

Former

Never

Diabetes

Yes

No

$60(93.8)$

Comorbidities

Heart disease

Hypertension

Chronic obstructive pulmonary disease

$1(1.6)$

TABLE 1: Characteristics of patients treated with cervical selective nerve root block

The measurements of the foraminal area between the two reviewers were averaged for the purpose of our analysis. The range of deviation between the two reviewers for each subject was -0.227 to $0.182 \mathrm{~cm}^{2}$, with an average deviation of $0.001 \mathrm{~cm}^{2}$. The reviewers were not significantly different in their measurements of foraminal area ( $\mathrm{p}=0.829$, paired t-test).

The average foraminal area of the side ipsilateral to injection $\left(0.154 \pm 0.118 \mathrm{~cm}^{2}\right)$ was 


\section{Cureus}

significantly smaller than the contralateral asymptomatic side, respectively $\left(0.224 \pm 0.113 \mathrm{~cm}^{2}\right)$ $(P=0.0001)$. Mean pre-procedural VAS score for all patients was $5.48 \pm 2.23$ (range: $1-10)$ and mean post-procedural VAS score (30 minutes after the procedure) was $1.91 \pm 2.65$ (range: $0-10$ ). The average improvement in VAS for all patients after injection was $3.58 \pm 2.68$ points. An excellent response to SNRB was defined as complete resolution of arm pain, a good response was defined as at least a $50 \%$ reduction in arm pain, and a fair or poor response was defined as a less than $50 \%$ reduction in arm pain or no response to injection [8]. Forty-three of the 64 patients had a good/excellent response to injection (67\%). Ten patients (16\%) had a fair response to injection, nine patients (14\%) had no response to injection, and two patients (3\%) reported higher VAS scores after injection.

Table 2 provides a summary of clinical outcomes based on the degree of foraminal stenosis. The overall range of foraminal areas was 0.0145 to $0.5005 \mathrm{~cm}^{2}$.

\begin{tabular}{|c|c|c|c|}
\hline Area range $\left(\mathrm{cm}^{2}\right)$ & $n$ & Mean VAS benefit & p-value \\
\hline $0.0145-0.1360$ & 35 & $3.80 \pm 2.52$ & \multirow{4}{*}{0.140} \\
\hline $0.1370-0.2575$ & 17 & $3.18 \pm 2.60$ & \\
\hline $0.2576-0.3790$ & 8 & $2.25 \pm 3.45$ & \\
\hline $0.3800-0.5005$ & 4 & $6 \pm 0.81$ & \\
\hline \multicolumn{4}{|c|}{$\begin{array}{l}\text { TABLE 2: Mean change in VAS score by degree of stenosis as measured by foraminal } \\
\text { area }\end{array}$} \\
\hline
\end{tabular}

The foraminal areas were arbitrarily divided into four equal ranges, with the majority of patients falling into the group with the smallest neuroforaminal areas. In the groups with the most stenosis, we observed a trend in VAS scores with those patients with the highest degree of stenosis (i.e., the smallest foraminal area), having the greatest response to injection. Despite this initial trend, Group 4, those patient's with minimal foraminal stenosis $0.379-0.5005 \mathrm{~cm}^{2}$ $(n=4)$, also reported a significant improvement in VAS score after injection. The level of benefit between the four groups was not statistically significant $(\mathrm{p}=0.140)$. None of the patients had been treated with oral methylprednisolone. Diabetes and smoking status were not statistically significant predictors of patient response to injection.

\section{Discussion}

Our results suggest that patients with severe neuroforaminal narrowing benefit from cervical SNRB. While our study involved only a small group of patients, our results did not support our initial hypothesis. We had anticipated finding a subset of patients with very small foraminal areas that would not respond SNRB. Our hypothesis was based on the idea that there would be a critical stenosis or foraminal area that was too small to allow adequate steroid/anesthetic penetration. In contrast, our results suggest that patients with very small neuroforaminal areas are likely to have an immediate response to injection while patients with mild to moderate stenosis are less predictable. Interestingly, we also observed four patients with minimal foraminal stenosis that reported a dramatic improvement in VAS scores $(6 \pm 0.81)$ after SNRB. An excellent response was consistently reported in all four patients. One hypothesis to explain the outcome for these patients is that neural foraminal narrowing may be occurring in the dynamic flexion/extension position, which is not assessed on the standard static supine MRI 
[9]. An alternative explanation would be that these four patients had a more chemical radiculitis because of a tear in the annulus and spread of inflammatory cytokines into the epidural space. This may explain their symptoms of radiculopathy without an appreciable disc herniation [10-11] and may explain why these patients experienced a favorable response despite minimal foraminal narrowing.

Cervical SNRBs are often used as both a diagnostic and a therapeutic adjuvant in the management of degenerative cervical spondylosis [12-13]. While there is still considerable debate about whether local anesthetic alone or a combination of local anesthetic and corticosteroids is more effective [14-19], SNRBs have been demonstrated to be a useful treatment option in patients with both degenerative cervical and lumbar spondylosis. Multiple authors have suggested that patients older than 50 years of age with radicular symptoms respond better to cervical epidural steroid injections, yet often anecdotal experience weighs heavily on patient selection [3-4, 20]. Although positive imaging findings suggestive of neuroforaminal compromise provide some guidance in patient selection [21], MRI findings have not been reliably shown to be predictive of a positive response to SNRB, and there is little or no literature on how cervical foraminal area correlates with patient response to SNRB.

The use of interlaminar and transforaminal epidural steroid injections in the lumbar spine have been well described in the literature [1-2]. Less literature is available regarding the efficacy of injections in the cervical spine [12-13,15], with only a few prospective randomized trials reported to date [14, 22]. Bush, et al. [22] prospectively studied 68 patients with symptoms of cervical radiculopathy treated with serial epidural or periradicular corticosteroid injections. At an average of 39 months follow-up, 76\% (48 patients) had resolution of radicular symptoms without any surgical intervention. More recently, Anderberg, et al. [14] prospectively reviewed a series of 40 patients with cervical radiculopathy that underwent SNRB with either a combination of corticosteroids and local anesthetic or local anesthetic alone. A three-week follow-up time point revealed no significant difference between the treatment groups. The authors suggest steroids may not provide any additional benefit in patients undergoing SNRB for symptoms of cervical radiculopathy.

MRI findings of cervical spondylosis are exceedingly common after the age of 50 years of age [23-24], and while it is common to have MRI findings of spondylosis without corresponding clinical symptoms, it is also common to encounter the opposite with minimal radiographic findings and strong subjective clinical complaints. Interpreting the MRI findings and correlating these to patient complaints can be challenging; however, Strobel, et al. [21] demonstrated that the most important MRI finding predictive of response to transforaminal epidural steroid injection is neuroforaminal involvement. They retrospectively reviewed the results of 93 patients treated with transforaminal epidural steroid injections to determine whether MRI findings could predict response to treatment. The authors assessed pre- and postprocedure VAS scores in patients treated with a combination of local anesthetic and corticosteroids and found that foraminal disc herniation $(P=0.034)$, foraminal nerve root compromise $(P=0.013)$, and the absence of spinal canal stenosis $(P=0.013)$ were statistically significant predictors of a positive response to transforaminal epidural steroid injection and portended a better response to treatment. The results of our study corroborate these findings. There are some inherent limitations with MRI. Image quality may be compromised by section thickness, decreased signal-to-noise ratio due to coil selection and technical parameters, partial volume averaging, and cerebrospinal fluid pulsation artifact; however, in a comparison of MR multi-echo data image combination sequence and CT myelography, Dorenbeck, et al. [25] found no statistical differences between these imaging modalities in the assessment of neuroforaminal or spinal canal narrowing.

\section{Conclusions}


Nerve root injections are an important treatment option in the management of patients with cervical radiculopathy. Although our study population represents only a small subset of patients, our results suggest that those patients with very small neuroforamina secondary to stenosis respond well to injection, while those patients with moderate to mild stenosis are less predictable in their response to injection. While larger prospective studies are needed to verify these results, measurement of neuroforaminal areas on MRI may represent a useful preprocedural technique to predict which patients with symptoms of cervical radiculopathy secondary to foraminal stenosis are likely to respond to selective nerve root injections.

\section{Additional Information}

\section{Disclosures}

Human subjects: Consent was obtained by all participants in this study. University of Utah IRB issued approval 53330. This New Study Application qualifies for an expedited review by a designated University of Utah IRB member as described in 45 CFR 46.110 and 21 CFR 56.110. The research involves one or more activities in Category 5 (published in 63 FR 60364-60367). The designated IRB member has reviewed and approved your study as a Minimal risk study on $12 / 13 / 2011$. Federal regulations and University of Utah IRB policy require this research protocol to be re-reviewed and re-approved prior to the expiration date, as determined by the designated IRB member. Animal subjects: All authors have confirmed that this study did not involve animal subjects or tissue. Conflicts of interest: In compliance with the ICMJE uniform disclosure form, all authors declare the following: Payment/services info: All authors have declared that no financial support was received from any organization for the submitted work. Financial relationships: Wilson Z. Ray declare(s) personal fees from Depuy/Synthes. WIlson Z. Ray declare(s) personal fees from Ulrich. Wilson Z. Ray declare(s) personal fees from Harvest. Wilson Z. Ray declare(s) personal fees and stock/stock options from LDRH. Wilson Z. Ray declare(s) stock/stock options from Zeiss. Erica F. BIsson declare(s) personal fees from nView. Erica F. Bisson declare(s) a grant and personal fees from PCORI. CSM Surgical Trial (site PI). Other relationships: All authors have declared that there are no other relationships or activities that could appear to have influenced the submitted work.

\section{Acknowledgements}

The authors thank Kristin Kraus, M.Sc., for editorial assistance in the preparation of this paper.

\section{References}

1. Vad VB, Bhat AL, Lutz GE, Cammisa F: Transforaminal epidural steroid injections in lumbosacral radiculopathy: a prospective randomized study. Spine (Phila Pa 1976). 2002, 27:11-16. 10.1097/00007632-200201010-00005

2. Benoist M, Boulu P, Hayem G: Epidural steroid injections in the management of low-back pain with radiculopathy: an update of their efficacy and safety. Eur Spine J. 2012, 21:204-213. 10.1007/s00586-011-2007-z

3. Ferrante FM, Wilson SP, Iacobo C, Orav EJ, Rocco AG, Lipson S: Clinical classification as a predictor of therapeutic outcome after cervical epidural steroid injection. Spine (Phila Pa 1976). 1993, 18:730-736.

4. Slipman CW, Lipetz JS, DePalma MJ, Jackson HB: Therapeutic selective nerve root block in the nonsurgical treatment of traumatically induced cervical spondylotic radicular pain. Am J Phys Med Rehabil. 2004, 83:446-454. 10.1097/00002060-200406000-00007

5. Slipman CW, Lipetz JS, Jackson HB, Rogers DP, Vresilovic EJ: Therapeutic selective nerve root block in the nonsurgical treatment of atraumatic cervical spondylotic radicular pain: a retrospective analysis with independent clinical review. Arch Phys Med Rehabil. 2000, 81:741-746. 10.1016/S0003-9993(00)90104-7

6. Derby R, Lee SH, Date ES, Lee JH, Lee CH: Size and aggregation of corticosteroids used for epidural injections. Pain Med. 2008, 9:227-234. 10.1111/j.1526-4637.2007.00341.x 
7. MacMahon PJ, Eustace SJ, Kavanagh EC: Injectable corticosteroid and local anesthetic preparations: a review for radiologists. Radiology. 2009, 252:647-661.

10.1148/radiol.2523081929

8. Anderberg L, Annertz M, Brandt L, Säveland H: Selective diagnostic cervical nerve root block-correlation with clinical symptoms and MRI-pathology. Acta Neurochir (Wien). 2004, 146:559-565. 10.1007/s00701-004-0241-4

9. Bartlett RJ, Hill CA, Rigby AS, Chandrasekaran S, Narayanamurthy H: MRI of the cervical spine with neck extension: is it useful?. Br J Radiol. 2012, 85:1044-1051.

10.1259/bjr/94315429

10. Marshall LL, Trethewie ER, Curtain CC: Chemical radiculitis. A clinical, physiological and immunological study. Clin Orthop Relat Res. 1977, 129:61-67.

11. Peng B, Wu W, Li Z, Guo J, Wang X: Chemical radiculitis. Pain. 2007, 127:11-16. 10.1016/j.pain.2006.06.034

12. Anderberg L, Annertz M, Rydholm U, Brandt L, Säveland H: Selective diagnostic nerve root block for the evaluation of radicular pain in the multilevel degenerated cervical spine. Eur Spine J. 2006, 15:794-801. 10.1007/s00586-005-0931-5

13. Lin EL, Lieu V, Halevi L, Shamie AN, Wang JC: Cervical epidural steroid injections for symptomatic disc herniations. J Spinal Disord Tech. 2006, 19:183-186. 10.1097/01.bsd.0000190558.13248.e1

14. Anderberg L, Annertz M, Persson L, Brandt L, Säveland H: Transforaminal steroid injections for the treatment of cervical radiculopathy: a prospective and randomised study. Eur Spine J. 2007, 16:321-328. 10.1007/s00586-006-0142-8

15. de Leon-Casasola OA: Transforaminal cervical epidural injections . Reg Anesth Pain Med. 2008, 33:190-191. 10.1016/j.rapm.2007.05.007

16. Manchikanti L, Singh V, Falco FJ, Cash KA, Pampati V: Evaluation of the effectiveness of lumbar interlaminar epidural injections in managing chronic pain of lumbar disc herniation or radiculitis: a randomized, double-blind, controlled trial. Pain Physician. 2010, 13:343-355.

17. Ng L, Chaudhary N, Sell P: The efficacy of corticosteroids in periradicular infiltration for chronic radicular pain: a randomized, double-blind, controlled trial. Spine (Phila Pa 1976). 2005, 30:857-862. 10.1097/01.brs.0000158878.93445.a0

18. Stout A: Epidural steroid injections for cervical radiculopathy. Phys Med Rehabil Clin N Am. 2011, 22:149-159. 10.1016/j.pmr.2010.10.007

19. Yang Y, Chen L, Gu YD, Hu SN, Zhang H: Long-term impact of transfer of phrenic nerve on respiratory system of children: a clinical study of 34 cases (article in Chinese). Zhonghua Yi Xue Za Zhi. 2006, 86:1179-1182.

20. Slipman CW, Lipetz JS, Herzog RJ, Vresilovic EJ: Nonsurgical treatment for radicular of pain of zygoapophyseal joint cyst origin: therapeutic selective nerve root block. Arch Phys Med Rehabil. 2000, 81:1119-1122. 10.1016/S0003-9993(00)59558-6

21. Strobel K, Pfirrmann CW, Schmid M, Hodler J, Boos N, Zanetti M: Cervical nerve root blocks: indications and role of MR imaging. Radiology. 2004, 233:87-92. 10.1148/radiol.2331030423

22. Bush K, Hillier S: Outcome of cervical radiculopathy treated with periradicular/epidural corticosteroid injections: a prospective study with independent clinical review. Eur Spine J. 1996, 5:319-325. 10.1007/BF00304347

23. Irvine DH, Foster JB, Newell DJ, Klukvin BN: Prevalence of Cervical Spondylosis in a General Practice. Lancet. 1965, 285:1089-1092. 10.1016/S0140-6736(65)92674-7

24. Kolstad F, Myhr G, Kvistad KA, Nygaard OP, Leivseth G: Degeneration and height of cervical discs classified from MRI compared with precise height measurements from radiographs. Eur J Radiol. 2005, 55:415-420. 10.1016/j.ejrad.2005.02.005

25. Dorenbeck U, Schreyer AG, Schlaier J, Held P, Feuerbach S, Seitz J: Degenerative diseases of the cervical spine: comparison of a multiecho data image combination sequence with a magnetisation transfer saturation pulse and cervical myelography and CT. Neuroradiology. 2004, 46:306-309. 10.1007/s00234-004-1175-5 